\title{
Oral lichenoid lesions and amalgam fillings
}

\author{
Do oral lichenoid lesions improve or resolve if amalgam restorations are \\ replaced with an alternative material?
}

\section{Issa Y, Brunton PA, Glenny AM, Duxbury AJ. \\ Healing of oral lichenoid lesions after replacing amalgam restorations: a systematic review. Oral Surg Oral Med Oral Pathol Oral Radiol Endod 2004; 98:553-565}

Data sources Medline (1966-2000), Embase (1980-2000) and the Cochrane Library (issue 2, 2000) were reviewed along with reference lists of existing relevant reviews.

Study selection Studies were included if they were randomised controlled trials (RCT), cohort or case-control; reported in English; and dealt with amalgam restoration replacement in people who had oral lichenoid lesions (OLL).

Data extraction and synthesis Data were extracted that related to: study design; patient characteristics; position and type of OLL; details of histological confirmation and of patch test results; the number of patients receiving replacement restorations; duration of follow-up; and healing. No meta-analyses could be carried out because of the heterogeneity of the studies.

Results In total, 14 cohort and five case-controlled studies, of variable quality and including a total of 1158 participants, met the criteria. Patient ages ranged from 23-79 years. In all, 636 of the 1158 people whose OLL was suspected to be related to their amalgam restorations had these replaced with alternative materials. Positive skin patch test (SPT) results for more than one mercury compound varied widely between studies from a low of $16 \%$ to a high of $92 \%$. Different criteria were used to select patients who needed their amalgam restorations replacing, and also for whether the contacting restoration or all amalgam restorations were replaced. Follow-up periods varied from 2-114 months. The proportion of individuals achieving complete healing varied from $37 \%$ to $100 \%$ although, in total, $15 \%$ of patients showed no improvement after replacement of their amalgam restorations.

Conclusions In some people who have OLL, lesions may be seen to improve or heal following replacement of amalgam restorations.
Address for correspondence: AJ Duxbury, Unit of Oral Medicine, University Dental Hospital of Manchester, Higher Cambridge Street, Manchester M15 6FH, UK.

\section{Commentary}

Given the high prevalence of amalgam restorations and the low number of reported adverse effects, amalgam is clearly a very safe restorative material. In a small number of susceptible individuals, however, OLL may develop on areas of the oral mucosa that are in direct contact with amalgam fillings. As with adverse effects caused by drugs or other materials, a causal link can only be established by removing the offending filling and demonstrating resolution or improvement of the lesion. By definition, therefore, 100\% of OLL caused by amalgam fillings should exhibit healing on replacement with a material that the patient is not sensitive to. This was not the conclusion of the review.

The problem is that OLL caused by contact with amalgam fillings (AC-OLL) are difficult to distinguish clinically or histologically from other OLL, or from idiopathic oral lichen planus (IOLP). OLL may be caused by contact sensitivity to a restorative material, a side effect of a systemic drug, or graft versus host disease: the patient's history may help to distinguish these. When attempting to distinguish between iOLP and AC-OLL, however, the bilateral, symmetrical lesion distribution of iOLP and the close physical relationship of AC-OLL to restorations is the only guide: in individuals who have fillings on both sides of the mouth this distinction may be difficult to make.

This problem confounds most of the studies in this review and undoubtedly accounts for the large variability in the results of the reviewed studies. This is compounded by the poor quality of the studies and lack of any RCT, noted by the review's authors. Furthermore, our understanding of these conditions and the role played by amalgam in their aetiology has changed considerably. Thus, patient selection criteria and study design have changed over time, which significantly affects the outcome and interpretation of studies. This is a particular issue for this review because it only covered studies performed up to 2000, despite being published in 2004 .

The question we should really be asking is, how do we accurately identify those lesions that would respond to amalgam replacement? A close topographical relationship between lesions and amalgam fillings appears to be the best predictor. ${ }^{1-5}$ Histology does not appear to be helpful. ${ }^{6}$

Although the review concluded that SPT was only of limited value in distinguishing AC-OLL, it was not designed to study this as its primary objective. Interpretation of the data was also confounded by the reasons given above, plus large variability in the SPT methodologies used. It is clear that SPT methodology for identifying ACOLL needs refining, particularly with regard to the allergens used. Typically, SPT are applied for $72-96 \mathrm{~h}$ but some patients develop responses to mercury or amalgam up to 14 days later. ${ }^{7}$ Since most studies in the review only checked responses after 24 or $48 \mathrm{~h}$, it is not surprising that SPT was found to be a poor predictor of lesion improvement in this review. In fact, more recent studies have shown that SPT can be a helpful adjunct in confirming the clinical diagnosis. One study found that a strong topographical association plus a positive SPT was the best predictor of lesion improvement with a number-needed-to-treat of 1.1 (the number of people who would need to be treated to result in one successful treatment). ${ }^{5}$ SPT alone will never be $100 \%$ accurate, however, because $3.2 \%$ of the general 
population are SPT-positive for mercury or amalgam, leading to false positive results, and false negatives may occur because some AC-OLL could result from the irritant or traumatic effects of amalgam restorations on the mucosa (the Koebner phenomenon), rather than allergy, or from sensitivity to the plague attached to the amalgam rather than the restoration itself..

\section{Practice points}

The physical proximity of lesions to amalgam fillings is the best guide to a causal relationship.

SPT may provide valuable supportive/ confirmatory information about the causal relationship and help identify alternative materials that the patient is not sensitive to.

OLL in close physical contact with amalgam fillings may improve or resolve following replacement or coverage (eg, crowning) with an alternative restorative material to which the patient is not sensitive.

It is not necessary to replace amalgam fillings that are not in direct contact with mucosal surfaces.

Rarely, patients may develop OLL to other restorative materials, particularly composite and gold restorations.

Amalgam replacement is not beneficial in the treatment of patients with idiopathic oral lichen planus or other types of OLL.

\section{Martin H. Thornhill}

Department of Oral \& Maxillofacial Medicine \& Surgery,University of Sheffield School of Clinical Dentistry,Claremont Crescent, Sheffield, UK
1. Dunsche A, Kastel I, Terheyden H, Springer IN, Christophers E, Brasch J. Oral lichenoid reactions associated with amalgam: improvement after amalgam removal. $\mathrm{Br}$ J Dermatol 2003; 148:70-76.

2. Issa Y, Duxbury AJ, Macfarlane TV, Brunton PA. Oral lichenoid lesions related to dental restorative materials. Br Dent J 2005; 198:361-366, 549,

3. Laeijendecker R, Dekker SK, Burger PM, Mulder PG, Van Joost T, Neumann MH. Oral lichen planus and allergy to dental amalgam restorations. Arch Dermatol 2004; 140:1434-1438.

4. Lopez-Jornet P, Camacho-Alonso F, Gomez-Garcia F, Bermejo Fenoll A. The clinicopathological characteristics of oral lichen planus and its relationship with dental materials. Cont Derm 2004; 51:210-211.

5. Thornhill MH, Pemberton MN, Simmons RK, Theaker ED. Amalgam-contact hypersensitivity lesions and oral lichen planus. Oral Surg Oral Med Oral Pathol Oral Radiol Endod 2003; 95:291-299.

6. Thornhill MH, Sankar V, Xu XJ, et al. The role of histopathological characteristics in distinguishing amalgam-associated oral lichenoid reactions and oral lichen planus. I Oral Pathol Med 2006; 35:233-240.

7. Koch P, Bahmer FA. Oral lesions and symptoms related to metals used in dental restorations: a clinical, allergological, and histologic study. J Am Acad Dermatol $1999 ; 41: 422-430$.

Evidence-Based Dentistry (2006) 7, 74-75.

doi: $10.1038 /$ sj.ebd. 6400430

\section{Key to evidence graphic used in the Evidence-based Dentistry Joumal.}

The graphic is based on the Centre for Evidence-based Medicine Levels of Evidence tables
www.cebm.netlevels_of_evidence.asp (see Evidence-based Dentistry 2003;4: p 17-18)

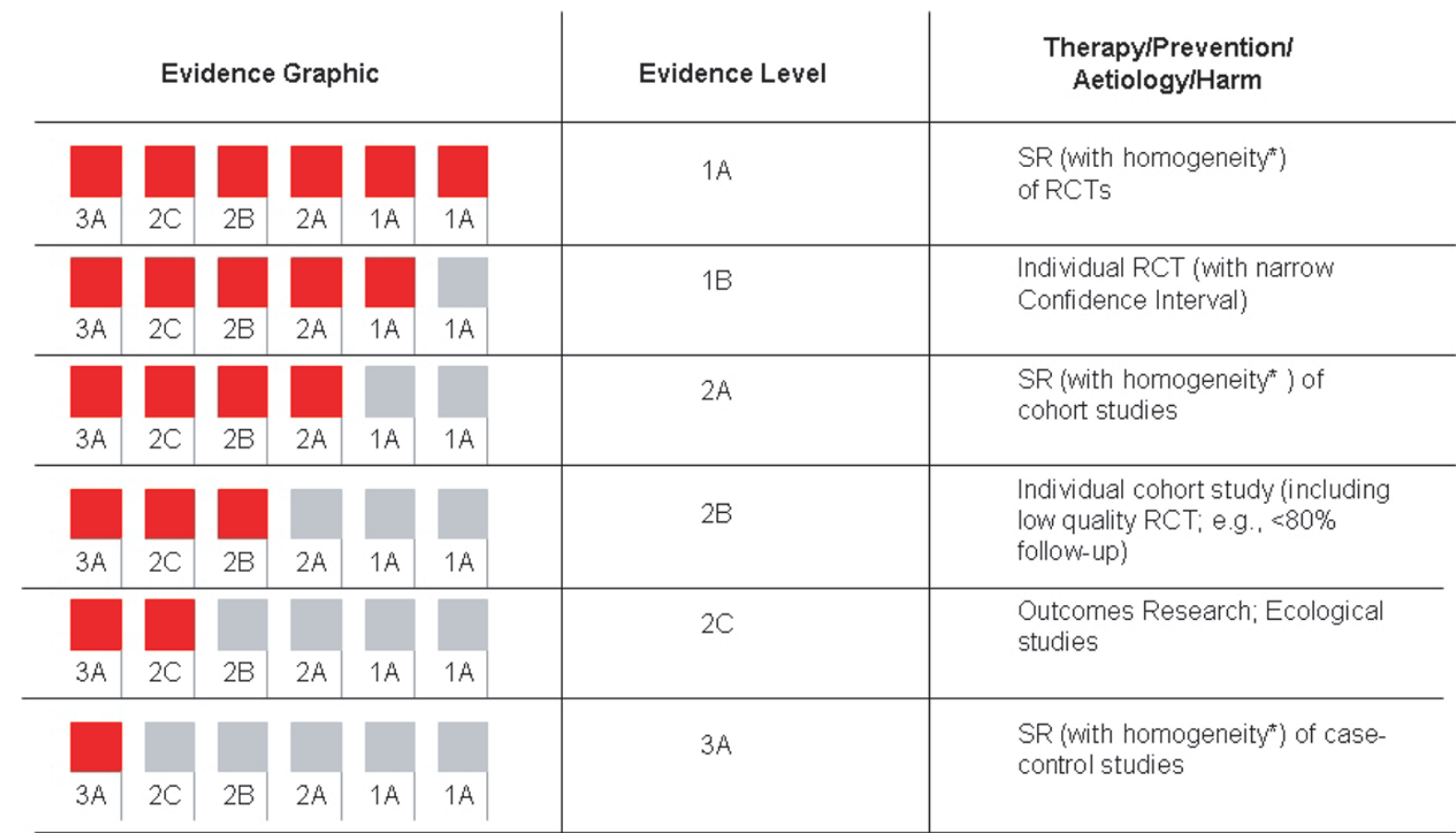

*By homogeneity we mean a systematic review that is free of worrisome variations (heterogeneity) in the directions and degrees of results between individual studies. Not all systematic reviews with statistically significant heterogeneity need be worrisome, and not all worrisome heterogeneity need be statistically significant. 